\title{
Agnès Guiderdoni-Bruslé, François Trémolières (textes réunis par), Littérature et spiritualité au miroir de Henri Bremond
}

Grenoble, Jérôme Millon, 2012, 218 p.

Daniel Vidal

\section{OpenEdition \\ Journals}

Édition électronique

URL : http://journals.openedition.org/assr/24002

DOI : 10.4000/assr.24002

ISSN : $1777-5825$

Éditeur

Éditions de l'EHESS

Édition imprimée

Date de publication : 30 décembre 2012

Pagination : 198

ISSN : 0335-5985

Référence électronique

Daniel Vidal, « Agnès Guiderdoni-Bruslé, François Trémolières (textes réunis par), Littérature et spiritualité au miroir de Henri Bremond ", Archives de sciences sociales des religions [En ligne], 160 | octobre-décembre 2012, mis en ligne le 14 mars 2013, consulté le 21 décembre 2020. URL : http:// journals.openedition.org/assr/24002 ; DOI : https://doi.org/10.4000/assr.24002

Ce document a été généré automatiquement le 21 décembre 2020.

(c) Archives de sciences sociales des religions 


\section{Agnès Guiderdoni-Bruslé, François Trémolières (textes réunis par), Littérature et spiritualité au miroir de Henri Bremond}

Grenoble, Jérôme Millon, 2012, 218 p.

Daniel Vidal

\section{RÉFÉRENCE}

Agnès Guiderdoni-Bruslé, François Trémolières (textes réunis par), Littérature et spiritualité au miroir de Henri Bremond, Grenoble, Jérôme Millon, 2012, 218 p. 
«Mais il s'agit bien de littérature! Notre curiosité voudrait percer le mystère déjà impénétrable de la zone littéraire, pour atteindre jusqu'à la vie intérieure de ceux qui ont lu et relu ces livres. Comprendre, admirer n'est rien; qui n'aimerait un tel sublime? Le vivaient-ils? Oui, je le crois ». Ainsi dit Henri Bremond: que son Histoire littéraire du sentiment religieux en France (désormais HLSR) n'est pas une mise en perspective de surplomb et globale de la spiritualité à travers les ouvrages des dévots ou des mystiques. Qu'elle ne s'entend pas comme glose à l'infini de textes et de témoignages, comme un verbe multiplié qui ferait effet «littéraire». Tout le contraire : aller s'il se peut au plus intime, et décisif, d'une expérience intérieure, en deçà/ au-delà d'une compréhension venue « du dehors » - car on ne « comprend » pas, à rigoureusement parler, le sublime - on s'y abîme de concert. Et sans doute y eut-il dès la parution des premiers tomes de cette Histoire une équivoque: s'agissait-il d'une galerie de portraits d'hommes et femmes pétris de dévotion, situés dans les plis d'une histoire de violences institutionnelles et de drames personnels ? Ou d'une appropriation par l'auteur des signes et symboles de spiritualités en éclats ou d'accomplissements mystiques - par quoi l'on pouvait, de ces témoignages écrits, à son tour s'en faire témoin, à sa propre guise les écrivant ? La récente réédition des onze tomes de l'HLSR (éd. J. Millon, 2006, 5 vol., dir. F. Trémolières) a permis de s'interroger à nouveaux frais sur les relations entre l'impératif "littéraire " et la posture « historienne» (Histoire et littérature chez Henri Bremond, J. Millon, 2009, dir. E. Fouilloux) et, dans le présent ouvrage, autorise une nouvelle évaluation des rapports entre spiritualité et littérature, dont la somme bremondienne ne cesse d'exposer l'indispensable tissage.

2 Suspendu a divinis pour avoir assisté en 1909 son ami mourant George Tyrrell, figure exemplaire du «modernisme », condamné par l'encyclique Pascendi, Bremond puise en Fénelon, dont il rédige à la même date l'Apologie, l'exemple d'une soumission qui maintient intacte la protestation d'une conscience convaincue de son droit, et va s'attacher, dans l'héritage du maître du pur amour, à l'univers de la mystique française. De cette marginalité doctrinale revendiquée, et sanctionnée, Augustin Laffay propose un passionnant dossier, qui ancre Bremond, et sa quête sans fin possible de la spiritualité, sur la tourmente d'une désaffiliation vécue comme illégitime. La dénonciation du modernisme, que l'Église considéra très vite comme danger mortel, concerna aussitôt l'écriture de Bremond, son «charme», ses résonances «mondaines», ses tournures et séductions. Et c'est au nom de ce même modernisme que la réception de son œuvre fut appréciée/dépréciée dans les milieux religieux, dont l'on peut prendre la mesure à travers les recensions des revues des différents ordres, présentées par Bernard Joassart en un "parcours bollandien ». Si l'on s'interroge sur le "métier » de Bremond - histoire, érudition, spiritualité - on souligne sa perspective d'« histoire des mentalités », et l'intention hagiographique qui la gouverne, dans cette quête des « expériences de ces enfants perdus de notre race, élancés vers le Bien sans 
ombre...». Tel critique la multiplication des exemples, mêlant hautes figures spirituelles et visages de peu de prix : "Où l'auteur devient insupportable, c'est dans cette avalanche d'inconnus [...] Est-ce là le gibier de littérature ? Sont-ce là sujets de grande histoire? ». Chef-d'œuvre littéraire, sans doute, mais « histoire vraiment solide et objective, non ». Il eut fallu choisir, entre le souci de ne rien perdre des expériences de tout un monde en proie à ce qu'Émile Goichot nomme "la faim de Dieu», et la rigueur de l'histoire comme ensemble qualifié de compétences, seule capable de rendre compte de l'unité ou de la diversité des modalités mystiques. Mais on voit que Bremond n'a cure d'obéir à cette obligation de choisir, précisément parce que nulle écriture n'est de hasard, et la sienne moins encore, qui accompagne le flot des spirituels dans leurs hésitations, leurs échecs, et la jouissance enfin de leur dieu. Et l'écriture qui s'ensuit est ainsi la respiration même de ces désarrois et de cette recherche d'une vision nue de Dieu. Le « modernisme » est bien ce privilège accordé à ce témoignage exceptionnel de l'intériorité chaque fois singulière en quoi s'énonce la spiritualité, ce "sentiment religieux » porté à sa plus haute tension. Écrire dès lors est bien, selon l'expression d'Henri Meschonnic, "n'avoir pas le choix », se déployer sous condition d'une nécessité sans recours. La somme de Bremond répond en toute clarté, qui put apparaître comme provocation, à cette injonction " poétique ".

3 La réception de l'œuvre à l'étranger s'inscrit au cœur de la polémique antimoderniste. Ainsi en Italie, où « l'expérience chrétienne de la modernité », selon Silvia Mostaccio, justifiait un nouveau style qui s'accordât avec la « réalité essentielle » de la spiritualité, cette épreuve attestée au plus profond de la subjectivité. Antonio Fogazzaro, dans son ouvrage Il Santo, mis à l'Index en 1906, fut en correspondance, et parfaite consonance, avec Bremond, partageant la nécessité de faire place aux "santi e poeti », témoins d'une quête personnelle de Dieu qui décide d'une configuration mystique échappant aux cadrages institutionnels. Ainsi des deux figures emblématiques, Dante et François d'Assise, « l'amoureux de la pauvreté et le poète des mondes supramondains ». Et Luigi Storzo, prêtre en exil, reconnaît en Bremond la passion d'une "grande mystique populaire ", qui permettait, selon Guiseppe De Luca, en son Introduzione à son Archivio italiana per la Storia della piétà, de " faire effraction profonde dans les études d'histoire ", et lire d'un œil entièrement renouvelé, l'immensité des textes, pour certains perdus de mémoire. Mais qui dit lecture nouvelle ne dit-il pas, aussitôt, neuve écriture de la passion de Dieu ? Si De Luca récuse sans doute cette relation immédiate d'un nouveau regard à une poétique singulière, et veut préserver la connexion entre théologie et dévotion, institution et spiritualité, il demeure cependant, au même titre que Bremond, en son fond questionné par «le problème de l'homme historique en rapport avec le mystère de son existence ». Cela même que la recherche déployée en l'HLSR se donne pour horizon absolu. Dans ses études sur Catherine de Sienne, Jacopo Passavanti, etc., Giovanni Getto dira plus nettement son accord avec la langue de Bremond, "cette poésie, écrit S. Mostaccio, engendrée par l'expérience mystique, en rapport avec un sentiment de l'indicible ».

4 La mise à l'Index en 1913 de la sainte Chantal de Bremond, se fondait sur des arguments doctrinaux, mais reprenait la critique du style, rappelle F. Trémolières. "Quant à la forme et au ton, écrivait J.-P. Lémius, l'un des rédacteurs du Pascendi, il suffit d'ouvrir l'œuvre pour que vous sentiez s'exhaler un esprit purement mondain, qui est en contraste strident avec le sujet ». Il en ira de même pour l'HLSR, qui connut même suspicion, mais ne fut pas condamnée. Définir le «sentiment » religieux suppose en effet que l'on s'adresse à l'émotion, ce movere coupable de substituer « la sincérité à la 
vérité du dogme, la conscience morale individuelle à l'enseignement moral de l'Église ». Du sentiment à l'expérience spirituelle, d'une qualité «naturelle » à un cheminement " surnaturel », écrire cette transition, qui vaut pour Bremond transfiguration, apparaît aux yeux des censeurs antimodernistes, "naturalisation de la foi ». Contre cet "immanentisme", qui dit le sacré comme expérience assujettie à l'histoire et la conscience singulières du spirituel, l'Église s'insurge, qui dénonce la «littérature » comme « le nouveau moyen de propagande moderniste».

Qu'entend-on par "expérience mystique»? demandait Jacques Le Brun, qui disait «improbable» sa définition. Le qualificatif peut paraitre contestable. Mais le « sentiment religieux » qui s'y rapporte, et la méthode d'histoire psychologique que Bremond mobilise soulèvent quelques difficultés qu'analyse Sophie Houdard. Concilier lecture psychologique et philosophie religieuse à partir d'un document littéraire revient à mettre en rapport d'écriture ce qui relève du sacré et ce qui relève du profane. À moins que l'on ne définisse le sentiment, avec François Marxer, dans sa contribution à la réédition de l'HLSR, comme "expression prenant en charge l'expérience et le discours impossible qui en rend compte». Bremond, au demeurant, n'en fait pas mystère : «Mon objet [...] est tout littéraire, descriptif et historique. Je raconte la vie des mystiques [...], je cite leurs pages maitresses, mais pour la discussion technique de leurs états [...] je m'en rapporte aux experts ». Aussi bien, S. Houdard peut-elle conclure que l'écriture bremondienne « documente une doctrine, mais peine à fournir les éléments d'une causalité ». Il reste que le "sentiment ", à coup sûr, est affaire de description, et le vécu concret qui s'y livre fonde l'expérience en une donnée de la conscience. Émile Goichot avait sans doute raison de dire que tel sentiment fleurait «le subjectivisme et l'immanentisme" - mais sans doute était-ce, pour Bremond, stratégie discursive : dire le moins pour avérer le plus; énoncer le plus humble pour faire valoir le sublime, cet « éclat insoutenable du divin (qui) nous plonge dans les ténèbres ", qu'ainsi qualifiait F. Trémolières dans son Fénelon et le sublime (ASSR, 152).

6 Bremond pouvait-il, de l'ensemble des textes et matériaux divers rassemblés en cette Somme littéraire et historique, ordonner assez ses lectures pour en proposer une synthèse capable de valoir connaissance exacte de la mystique en tous ses états ? Tout texte, en cette HLSR, est certes " monument ", remarque Agnès Guiderdoni-Bruslé et, à ce titre, conservant "la mémoire de l'expérience mystique", ne relève pas d'une lecture allégorique. Mais il est aussi bien «document», selon l'alternative posée par M. Foucault - et par là même "signe d'autre chose ", ouvert à l'interprétation de son dire et de sa "poétique ». De même en va-t-il de l'iconographie de l'HLSR, tableaux, épitaphes, frontispices, dont Pierre Antoine Fabre analyse la « transsubstantiation » qui s'opère de l'écrit dans l'image. Dinah Ribard accorde ainsi à l'usage par Bremond du document/monument bien plus qu'un statut de source: une fonction de "réceptacle encore riche de ce qui l'a empli». Source vive devrait-on dire, dont l'historien du sentiment religieux, par la tension de sa seule écriture, préserve la trace. De là, la capacité de cette écriture à déployer, à travers cette "forêt de témoignages", une " métaphysique de la prière ", doctrine sans doute, mais en laissant " parler tour à tour chacun en son nom » les maîtres retenus. Des «basses vallées de la vie dévote " aux «hautes montagnes mystiques»- de la "belle mort» de Catherine de Nivelles aux traités sur le pur amour, ce grand œuvre d'Alexandre Piny par exemple -, seule une écriture, en ce qu'Alain Cantillon nomme ses «marqueurs de style », peut autoriser la circulation des témoignages, et leur métissage. Plus encore : elle seule peut accéder à ce 
que Bremond appelle cette « zone indécise » où la simple dévotion se magnifie en haute spiritualité, et la mystique ne se décide que sur l'humilité d'une foi quotidienne. C'est à ce qu'un poète dirait "conjonction du corps et du jardin », que s'emploie l'écriture bremondienne, véritable espace littéraire où chaque phrase, écho d'un texte antérieur, est en même temps ouverture du texte à tout autre, d'identique horizon. Écriture intertextuelle, capable de libérer un "secret soustrait au discours", quand d'une "vallée » à une « cime " une même passion se joue. Question de style, on le voit, jamais clos en lui-même, mais ouvert au contraire à tout « dit » de spiritualité, et faisant de ces «dits» l'expression de l'«accord intime d'une sensibilité et d'une doctrine». Une " histoire littéraire " parait alors possible, dans cette cohabitation du texte et de son lecteur, celui-ci logé au cœur de celui-là, et le texte venu au plus intime de la conscience du lecteur. En ce partage des sensibilités et des verbes qui la disent et la font accéder à la rigueur d'une loi, le style de Bremond noue en un seul acte de connaissance ce qu'Alain Cantillon définit comme "posture langagière et posture spirituelle » qui, désormais, «ne sont plus qu'une ».

7 Mais il n'est, en cette écriture de la passion mystique, nul sens ultime, et nulle assomption de la quête. Le « style des saints ", que Bremond exalte dans le temps même où il l'énonce et le déploie en sa recherche d'offrandes spirituelles, toutes variations confondues, "marque ce point qui fuit à l'infini ». En ce sens, écriture et spiritualité participent d'une même "expérience de vie ", pour reprendre la formule d'Agnès Guiderdoni-Bruslé, où l'une et l'autre, indissolublement liées en la Somme de Bremond, portent témoignage d'une "saisie directe de la vie intérieure ", en ce qu'elle recèle de secret et d'incertitude, d'inconnaissance et de nuage - de raison mystique sans fin. 